\title{
Pulse confinement in optical fibers with random dispersion
}

\author{
M. Chertkov ${ }^{\dagger}$, I. Gabitov, and J. Moeser \\ Theoretical Division, Los Alamos National Laboratory, Los Alamos, NM 87545
}

Communicated by Leo P. Kadanoff, University of Chicago, Chicago, IL, September 19, 2001 (received for review April 26, 2001)

Short-range correlated uniform noise in the dispersion coefficient, inherent in many types of optical fibers, broadens and eventually destroys all initially ultra-short pulses. However, under the constraint that the integral of the random component of the dispersion coefficient is set to zero (pinned), periodically or quasiperiodically along the fiber, the dynamics of the pulse propagation changes dramatically. For the case that randomness is present in addition to constant positive dispersion, the pinning restriction significantly reduces average pulse broadening. If the randomness is present in addition to piece-wise constant periodic dispersion with positive residual value, the pinning may even provide probability distributions of pulse parameters that are numerically indistinguishable from the statistically steady case. The pinning method can be used to both manufacture better fibers and upgrade existing fiber links.

T he effect of random perturbations in optical fibers increasingly attracts attention as the demand for the quality of transmission grows daily $(1,2)$. The impact of randomness on signal transmission in a single-mode fiber is negative; it causes degradation of the signal and lowers transmission capabilities. In particular, amplifier noise (3-6) and random fiber birefringence (7-11) lead to random shifts in the pulse position (timing jitter) and to pulse broadening, respectively. Both effects eventually cause a destruction of bit patterns and lead to an increase of the bit-error-rate (BER), the most important parameter describing performance in fiber communications systems (12).

In the present paper, we consider the effect of random dispersion, which is, for ultrashort pulses, one of the major causes of bit-pattern destruction. However, we propose a way to significantly reduce the pulse deterioration and eventually reduce the BER caused by the noise in dispersion by using passive (independent of pulse properties) periodic control of the accumulated dispersion of the fiber link. The method may even provide statistically steady propagation of the pulse along the fiber and gives insight into general understanding of control mechanisms for nonlinear systems with randomness.

Chromatic dispersion is an important characteristic of a medium and can significantly degrade the integrity of wave packets. In practice, chromatic dispersion is not uniformly distributed and often exhibits random variations in space and time. On the other hand, wave propagation through the medium is usually much faster than temporal variations of the chromatic dispersion. Therefore, these random variations can be treated as "spatial" multiplicative noise that does not change in time. This multiplicative noise is conservative, and the wave energy remains constant during propagation through the medium. Recently, high-precision measurements of fiber chromatic dispersion as a function of a fiber length experimentally demonstrated the significance of the dispersion randomness $(13,14)$.

The overall chromatic dispersion in an optical fiber comes from two sources. The first source is the medium itself. The second source is the specific geometry of the waveguide profile. Material dispersion in the optical fiber is a relatively stable parameter, uniformly distributed along the fiber. However, waveguide dispersion is not nearly as stable. Existing technology does not yet provide accurate control of the waveguide geometry of modern fibers, where dependence of dispersion on wavelength is complex. As a result, the magnitudes of random variations of fiber chromatic dispersion are typically the same as, or in some cases even greater than, that of the mean dispersion $(13,14)$.

In the short-wavelength regime, a universal description of the signal envelope in the reference frame moving with the packetgroup velocity is given by the nonlinear Schrödinger equation for the complex scalar field, $\psi(z ; t)$, see for example ref. 12,

$$
-i \partial_{z} \psi=d(z) \partial_{t}^{2} \psi+2 \psi^{2} \bar{\psi}
$$

The equation is written in the dimensionless form. ${ }^{\ddagger}$ Variations in the medium enter Eq. 1 through the dispersion coefficient $d(z)=$ $d_{\text {det }}(z)+\xi(z)$, which is decomposed into its deterministic part, $d_{\text {det }}(z)$, and a random part, $\xi(z)$. Here $z$ is the position along the fiber and $t$ is the retarded time. The initial profile $\psi(0 ; t)$ is localized in $t$. We consider two different models of deterministic dispersion, both of which are standard in fiber optic communications. Model A is the case of constant dispersion, $d_{\mathrm{det}}=d_{0}$. In the absence of noise $[\xi(z)=0], \psi_{0}(z ; t)=a \exp \left[i z d_{0} / \mathrm{b}^{2}\right] \operatorname{sech}[t / b]$, where $a^{2} b^{2}=d_{0}$ ( $a$ is the peak amplitude, $b$ is the pulse width, $b$ is inversely proportional to bit-rate) is an exact soliton solution of [1]. The existence of the soliton (15) is the result of a dynamic equilibrium between dispersion and nonlinearity: the two spatial scales-nonlinearity, $z_{\mathrm{NL}}=1 / a^{2}$ and dispersion, $z_{d}=b^{2} / d_{0}$ coincide. Model B is the case of dispersion management (DM), $d_{\mathrm{det}}=d_{0} \pm d_{D M}(16)$. Here, dispersion is piecewise constant: positive and negative spans alternate with period $z_{D M}$, and $0<$ $d_{0}<d_{\mathrm{DM}}$. There is no exact solution for the pure (no noise) Model B, but theoretical evidence, confirmed by extensive numerical studies and experimental results, indicates the existence of a breathing solution (DM soliton) with a nearly Gaussian shape (17-20). The localized solution here is, again, due to the interplay of dispersion and nonlinearity. In the presence of a periodic dispersion map, however, the (DM) soliton acquires an important characteristic, quadratic phase (chirp). In contrast to conventional soliton solutions, DM solitons can exist for zero (or even negative) values of average dispersion.

Approximate scale characteristics of the dispersion noise present in real fibers can be extracted from experimental results $(13,14)$. These results show that the smallest scale of noticeable change in the dispersion value is approximately $\approx 1-2 \mathrm{~km}$. For

\footnotetext{
†To whom reprint requests should be addressed. E-mail: chertkov@lanl.gov.
}

${ }^{\ddagger}$ All parameters are presented here in dimensionless units that transform to real-world fiber units, according to the following rules. The envelope of the electric field is in the form $\psi=E / \sqrt{P_{0}}$, where $P_{0}$ is the peak pulse power. The propagation variable is $z=x\left(\alpha P_{0} / 2\right)$, where $x$ is distance along the fiber and $\alpha$ is the Kerr nonlinearity coefficient. The Kerr coefficient can be expressed in terms of other fiber parameters, $\alpha=2 \pi n_{2} /\left(\lambda S_{\text {eff }}\right)$, where $n_{2}$ is the nonlinear component of fiber refractive index, $\lambda$ is the operating wavelength, and $S_{\text {eff }}$ is an effective core area of the fiber. The spatial coordinate is $t=\tau / \tau_{0}$, where $\tau$ is in the reference frame of the group velocity, and $\tau_{0}$ is the characteristic pulse width. The dispersion coefficient is $d=2 \beta_{2} /\left(\alpha P_{0} \tau_{0}^{2}\right)$, where $\beta_{2}$ is the second-order dispersion parameter. The typical parameters for dispersion-shifted fiber are: $\lambda=1,550 \mathrm{~nm}, \tau_{0}=7.01 \mathrm{ps}$, $x=50 \mathrm{~km}, P_{0}=4 \mathrm{~mW}, \beta_{2}=2 \mathrm{ps}^{2} / \mathrm{km}, \alpha=10 \mathrm{Wt}^{-1} \mathrm{~km}^{-1}$.

The publication costs of this article were defrayed in part by page charge payment. This article must therefore be hereby marked "advertisement" in accordance with 18 U.S.C. $\$ 1734$ solely to indicate this fact. 
constant dispersion fibers (model A), the amplifier spacing is $\approx 50-60 \mathrm{~km}$, and for dispersion managed fibers (model B), the period of a typical dispersion map is also $\approx 50-60 \mathrm{~km}$. These scales are much longer than that of the dispersion variation, justifying the idealized consideration of ensemble averaging over short-correlated Gaussian noise for both models, according to the Central Limit Theorem. Previously, the stability of initial pulses in the presence of the short-range-correlated Gaussian uniform noise $\xi_{u}$ with zero mean, $\left\langle\xi_{u}\left(z_{1}\right) \xi_{u}\left(z_{2}\right)\right\rangle=D \delta\left(z_{1}-z_{2}\right)$, was studied for both models $\mathrm{A}$ and $\mathrm{B}$. The pulse-broadening effects of $\xi_{u}$ on both models A and B were studied numerically and by means of a variational approach (21-23).

We briefly discuss the dynamics in the limit of strong noise. On short scales, the nonlinearity is weak, and propagation is essentially linear: only the phase of the pulse changes because of rapidly varying dispersion, whereas its frequency spectrum varies slowly. In this weakly nonlinear case the transformation

$$
\psi(z ; t)=\int_{-\infty}^{\infty} d \omega \exp \left[-i\left(\omega t+\omega^{2}\left[\int_{0}^{z}\left(d\left(z^{\prime}\right)-d_{\mathrm{det}}\right) d z^{\prime}\right]\right)\right]
$$

from $\psi$ to $\varphi_{\omega}$ filters out fast oscillations. The evolution of $\phi_{\omega}(z)$ is given by

$$
\begin{aligned}
\left(-i \partial_{z}+i d_{0} \omega^{2}\right) \varphi_{\omega} & =2 \int d \omega_{1,2,3} \delta\left(\omega_{1}+\omega_{2}-\omega_{3}-\omega\right) \\
& \times \exp \left[-i \Delta \int_{0}^{z} d z^{\prime}\left(d_{\mathrm{det}}-d_{0}\right)\right] e^{-i \Delta \int_{0}^{z} d z^{\prime} \xi\left(z^{\prime}\right)} \varphi_{1} \varphi_{2} \bar{\varphi}_{3}
\end{aligned}
$$

where $\Delta \equiv \omega_{1}^{2}+\omega_{2}^{2}-\omega_{3}^{2}-\omega^{2}$. This equation describes the pulse propagation on longer scales. The term

$$
e^{-i \Delta \int_{0}^{z} d z^{\prime} \xi_{u}\left(z^{\prime}\right)}
$$

is a strongly fluctuating quantity whose ensemble average decays exponentially in $z$ for typical configurations of the noise $\xi_{u}$. For $\xi=\xi_{u}$, the ensemble average of the kernel is $\exp \left[-\Delta^{2} D z_{*} / 2\right]$, and $z_{*}$ is equal to $z$, so that the kernel is decorrelated at $z_{\xi}=$ $\left[\Delta^{2} D\right]^{-1} \sim\left[\omega^{4} D\right]^{-1} \sim b^{4} / D \sim 1 /(B R)^{4} * D$, where $B R$ is the bit-rate of the transmission system. The exponential decay of the kernel with $z$ disrupts the balance between nonlinearity and dispersion necessary for steady-pulse propagation; i.e., the natural noise in dispersion leads to the destruction of an initially localized signal.

The natural question is that of the existence of an artificial constraint capable of reducing and maybe altogether preventing this pulse broadening. We demonstrate that such a constraint does indeed exist and can be implemented readily in real fibers. All that is required is that the accumulated dispersion, $\int_{0}^{z} d y \xi(y)$ is set to zero, or pinned, either periodically or quasi-periodically with a period of the order of $z_{\xi}$ or less. The resulting Gaussian nonuniform noise, $\xi_{n}$ with zero mean is described by

$$
\left\langle\xi_{n}(y) \xi_{n}(z)\right\rangle=D\left(\delta(z-y)-\frac{1}{l_{j+1}-l_{j}}\right)
$$

if $l_{j}<y, z<l_{j+1}$; i.e., $y$ and $z$ belong to the same segment bounded by an adjacent pair of pinning points, $l_{j}$. Otherwise, there are no correlations. The pinning method improves pulse integrity independently of the particular shape of the pulse, and it works for both small and large levels of dispersion fluctuations. The improvement in propagation is achieved in a statistical sense: the change (in $z$ ) of ensemble-averaged correlation functions of various parameters characterizing a pulse is much slower if the pinning method is applied. The fundamental principal at the core of the method is to pin the difference between the accumulated value of actual dispersion and accumulated value of nominal dispersion to zero. In this sense, the pinning method is a generalization of the pure dispersion management technique (16-19), which consists of periodic compensation of the accumulated deterministic fiber dispersion by insertion of a compensating fiber with constant deterministic dispersion of an opposite sign. In relation to previous works on overcoming pulse deterioration caused by variation of dispersion in a fiber link, we stress that our technique is quite general. Our results are consistent both with experimental observations (14) for a longhaul propagation through a looped system with a complicated dispersion profile ${ }^{\S}$ and a guidanken numerical algorithm (24) for optimal ordering in the cabling process of the fiber spans of the same length but slightly varying constant dispersion."

In this article, we demonstrate the validity of the pinning method, first theoretically, addressing the weakly nonlinear case, and then by numerical extension of the analysis to the case of the moderate nonlinearity. Consider the effect of nonuniform noise, $\xi=\xi_{n}$, in the weakly nonlinear case. Pinning changes the correlation function for the noise, so that $z_{*}=z\left(l_{j+1}-z\right) /\left(l_{j+1}\right.$ $-l_{j}$ ) with $z$ from $\left[l_{j} ; l_{j+1}\right]$, where $l_{j}, l_{j+1}$ are pinning points. The ensemble average of the kernel is now a periodic (or quasiperiodic) function of $z$, and the kernel is a self-averaged quantity on $z$-domains longer than the pinning scale and shorter than the scale of nonlinearity. Thus, for $z$ large, the kernel's fluctuations are greatly suppressed, and [2] can be averaged over the fast fluctuations by simple replacement of $\varphi$ and the fluctuating kernel by their ensemble averages. In the case of Model A, additional averaging over a single pinning leg reduces the $z$-dependent kernel of to

$$
\exp \left[-\Delta^{2} D l / 4\right] \sqrt{\pi /\left[\Delta^{2} D l\right]} \operatorname{Erfi}\left[\sqrt{\Delta^{2} D l} / 4\right] .
$$

In the case of Model $\mathrm{B}$, one should average with respect to both the pinning period and the period of the periodic-dispersion map. The overall result of the averaging procedure is the emergence of a nonlinear term that does not vanish as $z \rightarrow \infty$. One also concludes that $z_{\xi}$ sets the critical pinning period: in the case of a strong noise, the pinning is efficient only if $l=l_{i+1}-$ $l_{i} \lesssim z_{\xi}$. It is possible to show that the averaged equation, valid only if pinning is applied, has a steady solution. The averaged equation approach is a priori applicable for a large but finite $z$, so the emergence of a steady localized solution for the averaged equation does not mean that the original problem possesses a steady-state. In the direct numerical simulation of Eq. 1, to which we now switch our attention, the effect is seen as essentially limiting the pulse broadening.

We perform numerical investigations of both models A and B with $\xi=\xi_{u}$ and $\xi=\xi_{n}$ in intermediate the case, $z_{N L} \sim z_{\xi}$. The initial $(z=0)$ pulse is the exact soliton for model $\mathrm{A}$, and it is a Gaussian pulse close to the respective DM soliton for model B. Fourier split-step scheme with $2^{13}$ temporal Fourier modes and

STo create a simulation of a long-haul experiment, a number of fiber spans were put together in (14). Each of the spans was constituted by the combination of different fibers to design a specific dispersion profile. The whole system was looped to simulate transmission over sizeable distances. The measurement of the values of the accumulated dispersion of each span, which were presumed to be the same initially, shows the presence of irregularity. It was shown that the insertion of an extra span compensating for this irregularity improves the transmission.

TPropagation of a pulse through a fiber with piece-wise constant dispersion was considered in (24). Dispersion of a single span, each of the same length, was taken to be a random Gaussian number with nonzero mean. It is shown that the propagation improves if the same sequence of spans is ordered in an alternating, descending order: the span with the largest positive value should be followed by the one with the largest negative value; the next pair is chosen according to the same principle from the bank of remaining spans, etc. 
periodic conditions imposed on the boundaries of the domain $t$ $\in[-180,180]$ is implemented. The spatial step is $z_{\text {step }}=0.01$, and the numerical convergence was checked by varying the size of the periodic box and the number of the Fourier harmonics. Parameters for the initial signal were chosen to be $d_{0}=1, a=1$ in model A, and $d_{0}=0.15, d_{D M}=0.1, z_{D M}=1,|\psi(0 ; t)|=0.79$ $\exp \left(-t^{2} / 2.6\right)$ in model B. The setup in model B is borrowed from (20) and corresponds to experimentally available DM fibers. Gaussian zero-mean noise correlated at $z_{n}=0.1$ with amplitude $d_{n}=1$ models the $\delta$-correlated uniform noise with $D=\mathrm{d}_{n}^{2} z_{n}=$ 0.1 . The nonuniform noise is constructed from the uniform noise by the following subtraction at every pinning leg:

$$
\xi_{n}(z)=\xi_{u}(z)-\frac{1}{l_{j+1}-l_{j}} \int_{l_{j}}^{l_{j+1}} d y \xi(y) .
$$

Pinning strategies of two types are considered: strictly periodic, $l_{j+1}-l_{j}=l$, where $l$ is fixed; and quasi-periodic, $l_{j+1}-$ $l_{j}=l(1+\eta)$, where $\eta$ is a random number uniformly distributed between $\pm 1 / 2$. The averaged (or otherwise strict) pinning period for the nonuniform case is taken to be 1,5 , or 10. The simulation runs until the pulse arrives at $z=95$. Statistics were collected for $10^{2}-10^{3}$, and, in a special case $10^{4}$, realizations.

The averaged pulse-width (full width at half-maximum amplitude) as a function of $z$ is shown in Fig. 1. The capital letter subscript of the figures corresponds to the type of model, A or B. Solid black represents uniform noise, and red, green, and blue represent nonuniform noise with pinning period $l$ equal to 1,5 , and 10, respectively. The quasi-periodic curves are dashed and of the same color as the respective periodic ones.

For Model A, all types of nonuniform noise demonstrate a significant reduction in the rate of pulse broadening when compared with the uniform case. The individual configurations that degrade (through pulse splitting, etc.) in the uniform case maintain pulse integrity when each type of nonuniform compensation is applied. The dependence on the pinning period is monotonic: the peak amplitude of the pulse decays faster as the pinning period increases. The difference between periodic and respective quasi-periodic cases is minor, with a slightly better confinement observed for the quasi-periodic case. The destruction of the pulse also is accompanied by emission of continuous radiation by the soliton. The radiation is seen clearly in the movie made for individual runs. Once the radiation reaches the boundaries of the box, it reflects and starts to interfere with the still-localized solution (at $z \geq 20$ ).

The effect of nonuniform noise is more dramatic in the case of Model B. For nonuniform compensation with the averaged period $l=1$ (and also less), one observes a tendency toward statistically steady behavior: the average pulse width does not decay (in contrast to a decay in the uniform case), and the probability distribution function of the pulse width (and of other variables characterizing the pulse propagation, such as amplitude) does not change shape with $z$. (Notice that for the case with the same pinning period $l=1$ but greater $D=2.5$, a minor but still observable degradation of pulse occurred. This change is consistent with the statement made in the text concerning the efficiency of the pinning: the greater is $D$, the lower is the critical pinning period.) There is no visible emission of radiation by the localized solution for any case of Model B. We also have checked that temporal and spatial averages (for example, for the PDF of the pulse width) coincide. The dependence on the type of

Movies of single-realization dynamics in $z$, a comparative plot of the dispersion profile with and without compensation, and more figures characterizing statistics of the pulse propagation in the various cases considered are available at http://cnls.lanl.gov/ chertkov/ Fiber.
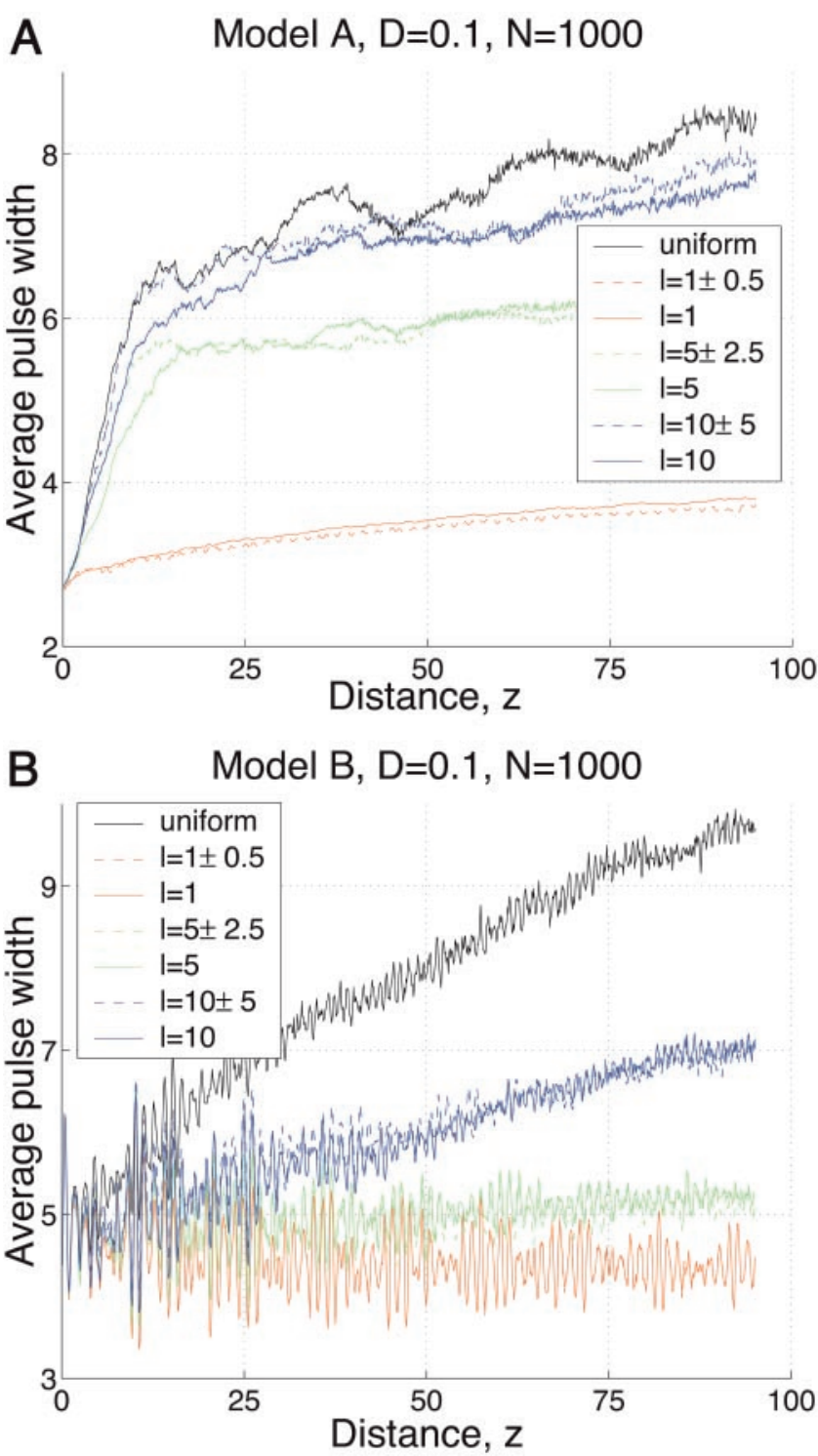

Fig. 1. Averaged pulse-width as a function of $z$ measured at the half-ofthe-peak-amplitude position. Fig. $1 A$ and $B$ correspond to the models $A$ and $B$, respectively.

compensation (for $l>1$ ) is monotonic, and the difference between the random and quasi-random cases is minor, as in the case of Model A. Notice that the tremendous reduction in the pulse decay is achieved by very minor changes in dispersion.

Our analysis gives practical recommendations for improving fiber system performance that is limited by randomness in chromatic dispersion. The limitation originates from accumulation of the integral dispersion. The distance between naturally occurring nearest zeros of the accumulated dispersion grows with the fiber length $(\sqrt{z})$. This growth causes asymptotic decay of the nonlinearity with $z$ and, therefore, pulse degradation. We have shown that the signal can be stabilized by periodic or quasi-periodic pinning of the accumulated random dispersion.** This result can be achieved by first measuring the mismatch between the nominal dispersion of the fiber line and the actual

**Real fiber spans, usually each of length $0.5-2 z_{N L}$, are not homogeneous, as they are often produced at different times by different companies. 
accumulated dispersion, and second, inserting a small fiber to compensate for this mismatch.

We are grateful to G. D. Doolen, G. Falkovich, I. Fatkullin, J. Hesthaven, I. Kolokolov, V. Lebedev, P. Lushnikov, P. Mamyshev, F. G. Omenetto, H. Rose, T. Schaefer, Z. Toroczkai, and S. Tretiak for constructive

1. Steinmeyer, G., Sutter, D. H., Gallmannn, L., Matuschek, N. \& Keller, U. (1999) Science 286, 1507-1512.

2. Thomas, G. A., Ackerman, A. A., Prunchal, P. R. \& Cooper, S. L. (2000) Phys. Today 53, 30-34

3. Elgin, J. N. (1985) Phys. Lett. A 110, 441-444.

4. Elgin, J. N. (1993) Phys. Rev. E Stat. Phys. Plasmas Fluids Relat. 47, 4331-4341.

5. Gordon, J. P. \& Haus, H. A. (1986) Opt. Lett. 11, 665-667.

6. Falkovich, G., Kolokolov, I., Lebedev, V. \& Turitsyn, S. (2001) Phys. Rev. E Stat. Phys. Plasmas Fluids Relat. 6302, 5601-5604.

7. Poole, C. D. (1988) Opt. Lett. 13, 687-689.

8. Poole, C. D. (1989) Opt. Lett. 14, 523-525.

9. Poole, C. D., Winters, J. H. \& Nagel, J. A. (1991) Opt. Lett. 16, 372-374.

10. Gisin, N. (1991) Opt. Comm. 86, 371-373.

11. Wai, P. K., Menyuk, C. R. \& Chen, H. H. (1991) Opt. Lett. 16, 1231-1233.

12. Agrawal, G. P. (1997) Fiber-Optic Communication Systems (Wiley, New York)

13. Mollenauer, L. F., Mamyshev, P. V. \& Neubelt, M. J. (1996) Opt. Lett. 21 1724-1726. comments. M.C. wishes to acknowledge the support of a J. R. Oppenheimer fellowship and the hospitality of the Institute for Theoretical Physics (Santa Barbara, CA), where part of this work has been done. This work was supported in part by Department of Energy Contract W-7405-ENG-36 and by a Department of Energy Program in Applied Mathematical Sciences Grant KJ-01-01 (to I.G.).

14. Mollenauer, L. F., Mamyshev, P. V., Gripp, J., Neubelt, M. J., Mamysheva, N., Gruner-Nielsen, L. \& Veng, T. (2000) Opt. Lett. 25, 704-706.

15. Zakharov, V. E. \& Shabat, A. B. (1972) Zh. Eksp. Teoret. Fiz. 34, 62-78.

16. Lin, C., Kogelnik, H. \& Cohen, L. G. (1980) Opt. Lett. 5, 476-478.

17. Gabitov, I. \& Turitsyn, S. K. (1996) Opt. Lett. 21, 327-329.

18. Gabitov, I. \& Turitsyn, S. K. (1996) JETP Lett. 63, 861-863.

19. Smith, N., Knox, F. M., Doran, N. J., Blow, K. J. \& Bennion, I. (1996) Electron. Lett. 32, 54-55.

20. Turitsyn, S. K., Schafer, T., Spatschek, K. H. \& Mezentsev, V. K. (1999) Opt. Comm. 163, 122-158.

21. Abdullaev, F. Kh., Caputo, J. G. \& Sorensen, M. P. (1998) in New Trends in Optical Soliton Transmission Systems, ed. Hasegawa, A. (Kluwer, Dordrecht, The Netherlands).

22. Abdullaev, F. Kh., Bronski, J. C. \& Papanicolaou, G. (2000) Physica D 135, 369-386.

23. Abdullaev, F. Kh. \& Baizakov, B. B. (2000) Opt. Lett. 25, 93-95.

24. Ohhira, R., Hasegawa, A. \& Kodama, Y. (1995) Opt. Lett. 20, 701-703. 